\title{
La calidad periodística mediante el análisis del discurso de los profesionales de la información
}

\author{
Mònica PARREÑo RABADÁN \\ Universitat de València \\ mopara@alumni.uv.es
}

Recibido: 7 de mayo de 2014

Aceptado: 10 de noviembre de 2014

\begin{abstract}
Resumen
La investigación indaga las opiniones de los periodistas sobre la influencia de los cambios en el campo periodístico en las últimas décadas, es decir, qué opinan los periodistas sobre lo que permanece, lo que ha desaparecido y aquello que es nuevo. La base teórica se fundamenta en la teoría del campo y del habitus de Pierre Bourdieu y en el análisis de la estructura comunicativa desde las perspectivas económica, sociológica, histórico-política y tecnológica. Se han aplicado una serie de métodos complementarios: análisis cualitativo de entrevistas en profundidad y análisis pragma-dialéctico de los argumentos de los periodistas entorno al concepto de calidad periodística ${ }^{1}$.
\end{abstract}

Palabras clave: calidad, periodismo, argumentación, pragma-dialéctica, periodistas.

\section{The quality of journalism through the analysis of the discourse of the professionals of the information}

\begin{abstract}
The research investigates the opinions from journalists on the influence of changes in the field of journalism in the recent decades, ie, what are the journalists' thoughts as regards the influence of what remains, what is being or has been destroyed and what is new in the field of journalism? The theoretical basis is based on the theory of field and habitus of Pierre Bourdieu and the analysis of the communicative structure from economic, sociological, historical, political and technological perspectives. I have applied complementary methods: qualitative analysis of in-depth interviews and pragma-dialectical analysis of the arguments of journalists around the term of quality of journalism.
\end{abstract}

Keywords: quality of journalism, argumentation, pragma-dialectical analysis, journalists.

\section{Referencia normalizada}

PARREÑO RABADÁN, Mònica (2015): "La calidad periodística mediante el análisis del discurso de los profesionales de la información". Estudios sobre el Mensaje Periodístico. Vol. 21, Núm. 2 (julio-diciembre), págs.: 1137-1147. Madrid, Servicio de Publicaciones de la Universidad Complutense.

Sumario: 1. Fundamentos de la investigación. 2. Análisis pragma-dialéctico del discurso; 2.1. Estudio empírico de la argumentación; 2.2. Reconstrucción de los argumentos. 3. Causas y consecuencias del descenso de la calidad periodística. 4. Referencias bibliográficas.

1 El campo periodístico y los profesionales de la información en el País Valenciano. Análisis de los argumentos de los periodistas sobre el periodismo es el título de la tesis internacional que defendí el pasado 9 de noviembre de 2012 en la Universitat de València, dirigida por el Dr. Rafael Xambó Olmos del Departamento Sociología y Antropología Social y el Dr. Jesús Alcolea Banegas del Departamento de Lógica y Filosofía de la Ciencia, ambos de la Universitat de València. La tesis fue calificada con Cum Laude, por unanimidad, y el tribunal estaba compuesto por el Dr. Hugh O’Donnell de la Glasgow Caledonian University, la Dra. Francisca Snoeck Henkemans de la University of Amsterdam y el Dr. Enric Casaban de la Universitat de València. La tesis se puede consultar en el enlace de Teseo: https://www.educacion.gob.es/teseo/mostrarSeleccion.do\# 


\section{Fundamentos de la investigación}

La incorporación de la esfera pública en el ámbito de la teoría de la argumentación tiene en cuenta el marco social donde la comunicación es entendida no como un requisito de conocimiento compartido, sino como una necesidad de estrategias comunes de representación que facilitan la acción cooperativa (Willard, 1989a: 166). Por tanto, se tiene que tener en cuenta el contexto donde se produce la actividad argumentativa, también cómo se genera y la interacción a la que da lugar. Según algunas aportaciones teóricas en el campo de la argumentación, ésta es vista como una construcción social: el argumento es una interacción que fluctúa en el tiempo y se beneficia tanto de la "estructura de la conversación" como de las "relaciones" y las "orientaciones", es decir, en el espacio de la intersubjetividad, el argumento es coherente no sólo por la fuerza ilocucionaria de los actos de habla (Willard, 1989b: 66).

En las últimas décadas, los estudios sobre lógica formal, centrados principalmente en las estructuras de los argumentos, las falacias y los criterios de evaluación y las conexiones con otros campos relacionados se han desarrollado, más allá de las primeras orientaciones pedagógicas y teóricas, hacia nuevas formas de aproximación práctica y social interrelacionadas con la lógica informal (por ejemplo, la lógica dialógica de Walton y Barth; la pragma-dialéctica de van Eemeren y Grootendorst; la comunicación del discurso de la escuela americana y los trabajos que conectan retórica y lógica informal de Wensel).

Para establecer un procedimiento de estudio de la argumentación y la percepción de los periodistas establecemos como punto de partida el análisis extradiscursivo, es decir, dar cuenta del habitus de los agentes periodísticos y la interrelación con el campo periodístico así como la incidencia del contexto en el discurso:

Estudiar cómo se ha estructurado la práctica periodística y las condiciones de objetivización del habitus periodístico.

Analizar los grupos que han contribuido a la producción de un campo simbólico más o menos institucionalizado con agentes especializados en la producción y distribución de noticias.

Esta primera contextualización es complementaria con el análisis intradiscursivo que muestra la incidencia que tienen en el campo y el habitus de los periodistas en la construcción de las retóricas sobre la profesión. Así, mediante el análisis cualitativo del discurso obtenido a través de las entrevistas en profundidad a los periodistas, se intenta responder a las preguntas de investigación que situaran el marco necesario para conocer los principios de la práctica periodística en un contexto determinado. De forma que el análisis del discurso más el conocimiento de la estructura social, económica, política e histórica permite la aproximación sociológica a la práctica periodística en un contexto determinado y a la percepción del campo por parte de los agentes que lo hacen posible.

La selección estratégica de dieciocho periodistas entrevistados es una muestra significativa y suficiente que recoge la diversidad de discursos, la representación de los medios de información más relevantes del País Valenciano, los diferentes ámbitos geográficos (Castellón, Valencia y Alicante). Además, para que la muestra sea lo más equilibrada posible, se han seguido también criterios de selección por género y por edades. 
Los objetivos metodológicos se corresponden con las preguntas de investigación cuyo esquema de las correspondencias se establecen como gráfica del diseño de la investigación y de los métodos escogidos (Parreño, 2012).

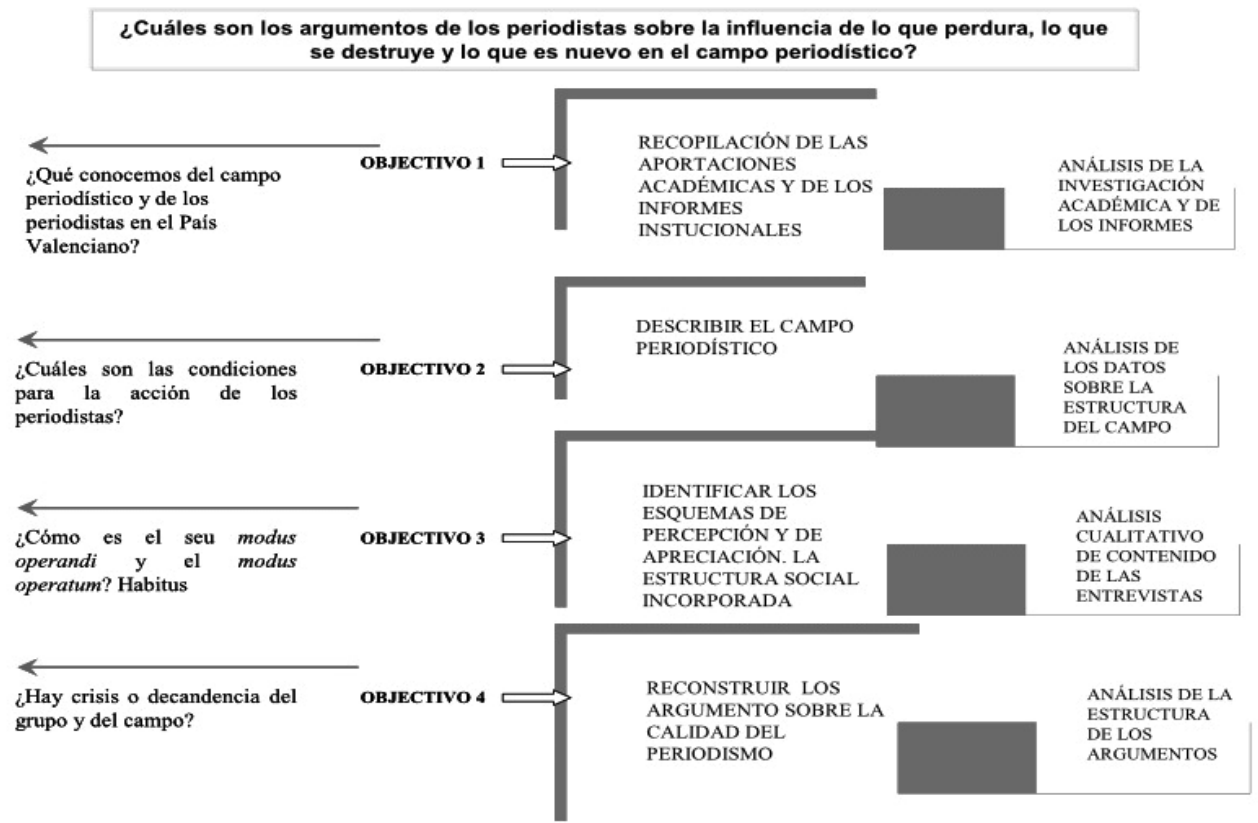

Gráfico 1: Objetivos de investigación y complementación de métodos (elaboración propia)

Los fundamentos teóricos de la investigación están basados en la teoría del campo del sociólogo francés Pierre Bourdieu para el análisis de la estructura comunicativa, el campo periodístico y el habitus de los periodistas. Esta teoría se amplia con el uso de la teoría de la argumentación, en su parte empírica, para conocer en el marco citado el debate y los argumentos de los periodistas sobre el concepto de calidad en el periodismo. El uso de las entrevistas en profundidad y el consiguiente análisis cualitativo de las mismas aporta una comprensión extensa del discurso, opiniones y reflexiones de los periodistas. Con este material analizado se detectan los comentarios sobre la calidad periodística que serán analizados posteriormente siguiendo la técnica de análisis de argumentos, llamada pragma-dialéctica. Por supuesto, se podría analizar cualquier concepto relevante siguiendo el mismo procedimiento. Por tanto, se utiliza el análisis cualitativo de contenido de las entrevistas desde dos perspectivas: sociológica y argumental. Ambas perspectivas se nutren mutuamente para darnos a conocer aspectos más variados y amplios de la percepción de los profesionales de la información del campo periodístico.

Se ha seguido, en definitiva, una estrategia de complementación de métodos (Bericat, 1998) y, por tanto, una analítica multimétodo para intentar aproximar la compleja realidad al conocimiento científico. 
El análisis del campo, según Bourdieu, consiste en el análisis de las estructuras sociales externas y de las relaciones de los espacios estructurados de posiciones, susceptibles de ser analizados al margen de la posición que ocupen los agentes en el campo. Por otra parte, los campos son dinámicos por su constante definición y redefinición de las relaciones de fuerza entre los agentes y las instituciones en juego, a los largo de la historia del campo, y también por la definición de sus límites en cuanto a su autonomía, relativa respecto a otros campos.

En el primer caso, un ejemplo es el desafío a la propia estructura del campo de aquellos que se acaban de incorporar al mismo, los más jóvenes que provocan un subversión del capital del campo. En el caso del periodismo se ha producido una brecha generacional por la introducción de las tecnologías de la información y la ausencia de profesionales experimentados porque, a causa de la crisis, han sido despedidos o prejubilados. En este cambio estructural del campo periodístico, la transmisión del savoir faire de la profesión se ha suprimido prácticamente. En cuanto al dinamismo y la autonomía, Bourdieu consideraba que el campo periodístico es dependiente del político y del económico y sujeto, por tanto, a las exigencias y demandas de éstos.

El habitus, entendido como una "disposición reglada para engendrar conductas regladas y regulares fuera de toda referencia a las reglas, porque es una conducta reglada sin obedecer a ninguna regla" (Bourdieu, 1988: 72). De forma que las acciones de los agentes son explicadas por las disposiciones adquiridas en el habitus. La lógica de la práctica en el campo permite que los miembros de un mismo grupo, provistos del mismo habitus, dispongan de unos códigos que les permiten relacionarse con espontaneidad, resolver conflictos y superar dificultades: el código compartido asegura una comunicación mínima. Pero una cosa es la lógica (el código) y otra los esquemas prácticos de la lógica parcial o blanda que tienen una coherencia parcial, que no pueden ser objetivados ni clasificados como lo hacen los etnólogos, porque pierden su propia lógica. El habitus contiene la historia trasmitida por el grupo y encarnada en los agentes que de esta forma producen los actos dotados de una lógica práctica y apropiados a las situaciones, hecho que produce una ilusio al convertir la necesidad en virtud. De forma que la historia incorporada en el habitus del grupo y de los agentes produce una homogeneidad que facilita la objetivación de la práctica.

\section{Análisis pragma-dialéctico del discurso}

Las fuentes de inspiración teórica de la pragma-dialéctica provienen del racionalismo crítico de Karl Popper, de la lingüística pragmática y de la lógica dialógica (de ahí la denominación de pragma-dialéctica). También recibe influencia de la teoría de los actos de habla de Searle, la lógica de Grice del discurso ordinario, la lógica dialógica de Lorenzen y la dialéctica formal de Barth and Krabbe (Eemeren, et. al., 1996: 274).

\subsection{Estudio empírico de la argumentación}

El estudio empírico de la argumentación trata de la reconstrucción, el análisis y la evaluación de los argumentos. La aproximación a la teoría de la argumentación de la pragma-dialéctica o pragma-dialectical approach, desarrollada por los académicos Frans H. van Eemeren y Robert Grootendorst de la University of Amsterdam (1994, 
2004), trata de establecer la relación entre la dialéctica y la retórica con el objetivo de establecer un modelo ideal de discusión crítica. No obstante, el interés por el análisis de casos reales ha ido incorporando estrategias para la investigación de la argumentación en el lenguaje ordinario y el discurso que permiten la reconstrucción y la interpretación de los argumentos en consideración al contexto físico, situacional e institucional para decidir cuál es la interpretación más plausible (charitable interpretation) (Snoeck Henkemans, 1997: 104).

El modelo ideal normativo de la pragma-dialéctica en la discusión crítica conforma una serie de reglas y pasos que la regulan. Las reglas son necesarias para conseguir los objetivos de la discusión crítica y asumen el carácter pragmático de la argumentación. La teoría de la argumentación, desde la perspectiva de la pragma-dialéctica, permite el análisis de los argumentos en contextos de discusión o enfrentamiento de diferentes puntos de vista. De acuerdo con van Eemeren y Grootendorst (Eemeren et al., 1994: 69) la argumentación es parte de un diálogo, implícito o explícito, en el que una parte intenta convencer a la otra de la aceptabilidad de su punto de vista. El modelo se define como un esfuerzo que vincula la lógica con la argumentación (Woods, 2006: 304). Los argumentos, en la resolución de la diferencia de opiniones, aparecen en los diferentes pasos donde juegan un papel relevante los procedimientos de requerimientos (directivos, usos declarativos) y donde los diferentes movimientos son aceptados o no, o permitidos, según el paso en el que se produzcan. Una de las virtudes del modelo es su aplicación empírica, es decir, destaca la integración de las dimensiones normativa y descriptiva y el carácter instrumental del análisis de los diferentes tipos de actividad comunicativa.

La argumentación, tal y como la define van Eemeren, siempre trata sobre la producción de efectos, como convencer a un crítico razonable de un cierto punto de vista o investigar la fuerza de la convicción del argumento (Eemeren, Grotendorst, 2004). En este sentido también nos interesa el concepto de contexto tal y como es utilizado en el modelo pragma-dialéctico de análisis reconstructivo del discurso que hace posible los movimientos argumentativos implícitos en el mismo y que son pertinentes para la resolución del proceso argumental. En esta reconstrucción, se distinguen tres fuentes: el texto del discurso, el contexto (micro-contexto, meso-contexto y macrocontexto referidos al contexto lingüístico, el contexto macro-lingüístico y el contexto intertextual respectivamente), una tercera fuente que son las inferencias pragmáticas basadas en el sentido común (por ejemplo, inconsistencias del discurso). También se contempla en la reconstrucción la background information o el conocimiento de ciertas reglas o regulaciones, así como la información experta sobre un tema o campo concreto (Eemeren, 2011: 143-145).

\subsection{Reconstrucción de los argumentos}

El análisis de contenido cualitativo de las entrevistas en profundidad puede enriquecerse con la investigación de la argumentación de los periodistas sobre la autorreflexión de la práctica periodística y los valores defendidos para ser contrastados con los datos referenciales obtenidos del análisis cuantitativo de la estructura comunicativa, a su vez, la profundización en la argumentación visibiliza el contraste entre la inte- 
riorización de la autorreferrencia, es decir, los valores y principios que deberían ser, y la práctica que se lleva a cabo. El concepto de calidad periodística, indicador del estado actual de la práctica, que según los datos de la estructura comunicativa ha decrecido considerablemente durante las últimas décadas, funciona en la estrategia interpretativa de los argumentos de los periodistas sobre su percepción de la práctica como punto de vista de partida para conocer las causas y consecuencias del decrecimiento.

En las entrevistas en profundidad en las que preferiblemente se trata de facilitar la confianza al entrevistado necesaria para que su discurso fluya, es decir, casi podría ser un monólogo en el que surgen las dudas, la reflexión olvidada y donde el investigador tiene que actuar como un orientador sutil, la reconstrucción de los argumentos puede complementar el análisis de contenido cualitativo y ayuda a profundizar en los temas o conceptos generales. Los problemas relacionados con la calidad periodística aparecen en los pensamientos y creencias de los periodistas cuando hablan de su profesión.

Según esta estrategia de reconstrucción de los argumentos (maximally argumentative interpretation) (Eemeren, et al., 2002), y de acuerdo con la actitud comprensiva del analista (charitable analysis), es posible interpretar como argumentación una explicación o una observación, es decir, cuando la función argumental no es del todo evidente, pero puede estar implícita. Esta estrategia minimiza el riesgo de obviar posibles argumentos necesarios para la discusión. También permite analizar casos donde no sea posible ver claramente otra interpretación de significado o cuando el punto de vista interpretado no pueda ser un sin sentido.

Aplicamos el análisis y reconstrucción de los argumentos según la perspectiva pragma-dialéctica. Según esta perspectiva, cada premisa es considerada de forma independiente y suficiente, si es válida para dar apoyo al argumento principal. A través de los argumentos se manifiestan las diferencias de opinión en el paso de la argumentación. El objetivo es el análisis de la estructura para la investigación de los argumentos que no aparecen bajo estructuras evidentes, para ver las relaciones entre los argumentos y dar cuenta de las razones en el conjunto de las opiniones y esclarecer los argumentos que permanecen implícitos. En la reconstrucción dialéctica del discurso argumental, el analista busca las características que son fundamentales para resolver la discusión. La reconstrucción se guía por la búsqueda de los puntos clave del problema, las diferentes posiciones, los argumentos implícitos y explícitos, la estructura de los argumentos de cada parte (Eemeren et al., 1993: 60).

El ejemplo que facilitan los autores muestra que, en contextos de desacuerdo, la opción de interpretar máximamente es una herramienta útil para desvelar lo que está escondido y es imposible de ver según la lógica formal.

La cuestión del orador es en principio absurda, porque se entiende que las personas generalmente no quieren mojarse. Parece que el orador quiere decir alguna cosa más, como "si no coges el paraguas, te mojarás" o "la única manera de evitar mojarte es coger el paraguas". Para la estrategia de interpretación máximamente de los argumentos, la cuestión del orador es tomada como un argumento desde su punto de vista: "Sería mejor que cogieras un paraguas". 
Las estructuras de la argumentación se dividen en sencillas y complejas. El argumento sencillo (single argument) es la forma más simple y consiste en sólo dos premisas, normalmente una de ellas es implícita. La defensa de una proposición que manifiesta un punto de vista (standpoint) está compuesta por diferentes argumentos sencillos que forman una argumentación compleja. La estructura compleja está formada por más de dos premisas. La estructura de la argumentación se entiende cuando la complejidad de la argumentación se puede romper en diferentes argumentos sencillos, de acuerdo con la pragma-dialéctica, que son equivalentes a una razón para cada argumento sencillo (Snoeck Henkemans, 2001: 126-17).

La argumentación múltiple (multiple argumentation) consiste en el uso de más de un argumento para defender un punto de vista. Se distingue de la coordinativa porque cada argumento es suficiente fuerte como para soportar el punto de vista individualmente. Contrariamente, en la argumentación coordinativa cada argumento necesita apoyo en las otras premisas que le acompañan porque no son suficientes para defender el punto de vista. La argumentación subordinada se da cuando cada argumento da apoyo al argumento anterior y responde a la causa de por qué da ese apoyo o a la cuestión de cómo se sabe que da ese apoyo.

\section{Causas y consecuencias del descenso de la calidad periodística}

Cada fragmento seleccionado es analizado a partir del punto de vista siguiente: "la calidad en el periodismo ha decrecido a lo largo de las últimas décadas" (Parreño, 2012, 2013). Teniendo en cuenta las causas, es posible sistematizarlas como los principales problemas relacionados con la calidad y por extensión con el periodismo. Las principales causas, según la reconstrucción de los argumentos en defensa del punto de vista, se organizan por tópicos generales: precariedad profesional, crisis económica, adaptación a las tecnologías de la información (en adelante TIC), brecha generacional, homogeneidad de contenidos, imprecisión, inmediatez, pérdida de valores.

La principal consecuencia de los cambios que ha vivido el periodismo en las últimas décadas es el descenso de la calidad periodística. La calidad se ve influenciada por el entorno ya que la falta de libertad de expresión (Xambó, 2012) por el dominio del poder económico y financiero influye no sólo en el estado de la democracia sino también en los mecanismos que ésta utiliza para que los ciudadanos tengan información rigurosa y plural para decidir.

Según Gómez Mompart el periodismo actual no puede obviar la interrelación de lo local con lo global y debe trabajar en este sentido convirtiendo la información en "glocal": "el terreno más fértil a medio plazo del periodismo de calidad apunta más allá de un periodismo de simples noticias que, sin renunciar a ellas, aborde noticias complejas, informaciones en profundidad, temas "glocales", como resultado de un trabajo conjunto entre profesionales y expertos (Gómez Mompart, 2013: 237).

En el siguiente gráfico se esquematizan causas y las principales razones, extraídas de la reconstrucción de los argumentos que esgrimen los periodistas en relación al punto de vista sobre el decrecimiento de la calidad periodística. 


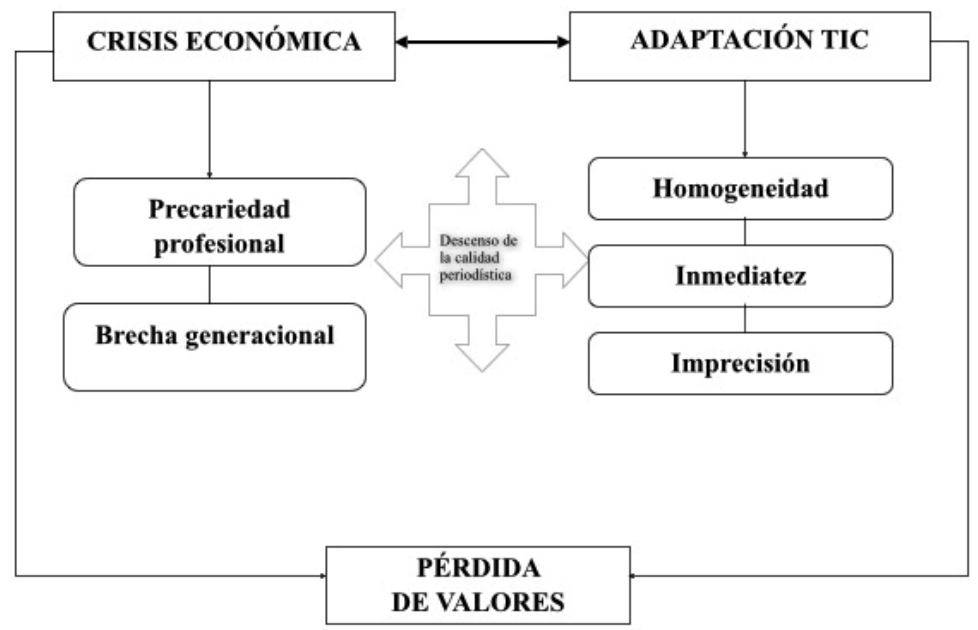

Gráfico 2: Relación de causas del descenso de la calidad periodística (elaboración propia)

Dos causas principales que agrupan al resto, una externa al campo y otra estructural o interna, a saber, la crisis económica y la adaptación a las TIC. Ambas están conectadas porque la adaptación a las TIC necesita recursos económicos que no están disponibles por la recesión. Sin embargo, ambas circunstancias están ocurriendo al mismo tiempo y es posible que los rápidos cambios producidos por la tecnología no sólo necesiten un esfuerzo de adaptación de los modos de producción sino que ésta se ha visto forzada por la crisis.

La crisis económica afecta directamente a las condiciones laborales de los profesionales $\mathrm{y}$, a su vez, ha producido la brecha generacional porque las empresas están contratando personal todavía no cualificado o becarios y despidiendo a los profesionales experimentados, por tanto, parte del conocimiento y experiencia que habitualmente era transmitido por los veteranos se pierde y los controles de calidad disminuyen. La práctica periodística relacionada con los modos de producción ha cambiado con la introducción de las TIC que han acelerado el sentido de inmediatez, propio del periodismo. También el problema de la homogeneidad de los contenidos y de la imprecisión del producto final es consecuencia de la falta de tiempo y de la competitividad.

Estas causas generales producen otra que implica a la totalidad de la profesión, a saber, la pérdida de valores y de prestigio, fundamentos de la credibilidad de la profesión. La siguiente figura muestra la interrelación entre las razones y las causas de la baja calidad periodística.

La crisis económica revela la dependencia del campo periodístico respecto del económico que, a su vez, produce mayor servilismo del campo político por la necesidad de recursos provenientes de las instituciones públicas (publicidad, subvenciones). No obstante, esta situación es similar a la de otros campos sociales, como el académico o el cultural, pero no explica suficientemente el cambio de tendencia del periodismo, es decir, la falta de precisión del contenido, la banalidad, la dependencia de las redes 
sociales y la ausencia de periodismo de investigación. El excelente trabajo de recopilación de estudios sobre la calidad periodística, los retos e investigaciones, coordinado por Gómez Mompart, Gutiérrez Lozano y Palau Sampio (2013) deja de manifiesto las dificultades epistemológicas para abordar el estudio de la calidad y también deja de manifiesto que ésta puede ser la clave de la supervivencia del periodismo como servicio al ciudadano en un espacio democrático.

Por otra parte, los argumentos de los periodistas sobre el bajo coste de producción de las noticias está relacionado con los salarios mínimos, es decir, la devaluación de la profesión y su prestigio, que produce contenidos de baja calidad, pues la mayoría coincide en la opinión de que es imposible producir contenido de calidad por profesionales con poca experiencia y mal remunerados. Los más experimentados y expertos no están tampoco preparados para el requerimiento del profesional polivalente del nuevo paradigma de producción.

Ambas causas de la baja calidad del periodismo están relacionadas o directamente producen la pérdida de valores. El periodismo es ante todo una profesión ideológica (Deuze, 2005: 443), aunque actualmente el periodista vigilante del poder se está sustituyendo por el periodista multimedia y prevalece la baja producción del periodismo de investigación. El punto de partida es la consideración de que toda introducción de nuevas formas de producción y venta requiere un tiempo de adaptación que, forzado por la crisis económica, ha desencadenado problemas para trabajar con rigor y precisión. Los valores son para los periodistas principalmente el cumplimiento de las reglas profesionales: contraste de noticias, neutralidad, rigor y honestidad (Deuze, 2002: 6). Los argumentos de los periodistas coinciden en este punto, de forma que revelan la asimilación de la lógica interna del campo o habitus. Sin embargo, la pérdida de valores también revela otro factor condicionante, a saber, la determinación de las condiciones estructurales internas del campo para el profesional.

El ciudadano ha ganado espacio de información (pluralidad de canales y plataformas de acceso rápido y fácil a la información), mientras que el campo del periodismo se enfrenta al reto de vivir de acuerdo con la demanda y exigencia del público y al tiempo ofrecer y proporcionar contenido de alta calidad. Pero este reto no se puede cumplir si las condiciones para la supervivencia están estrechamente ligadas a las esferas económica y política. Por tanto, debemos explorar la dinámica, a saber, el establecimiento de las relaciones de poder entre los actores e instituciones involucradas. También hay que definir y evaluar la percepción del campo tal y como se ve por las generaciones que han sido recientemente incorporadas, y los que están en la situación más precaria. Cuando los periodistas entrevistados han expresado su optimismo sobre el futuro, afirman la necesidad de que el campo sea gestionado por profesionales cualificados, ya que éstos son los que saben cómo escoger, escribir y difundir los contenidos bajo ciertos criterios y reglas aprehendidos. Por tanto, el objetivo de servicio y el cumplimiento profesional se justifica por la necesidad de mantener al público informado con rigor. La demanda de esta información por parte de los ciudadanos es importante para poder exigir esa calidad, rigor y autonomía a los responsables y agentes del campo periodístico. El estudio de esta demanda con herramientas etnográficas es otro de los retos que tenemos que afrontar. 


\section{Referencias bibliográficas}

BERICAT, Eduardo (1998): La integración de los métodos cuantitativo y cualitativo. Barcelona, Ariel.

BOURDIEU, Pierre (1988): Cosas dichas por Pierre Bourdieu. Barcelona, Gedisa.

DEUZE, Mark (2005): "What is journalism? Professional identity and ideology of journalists reconsidered". Journalism, 6 (4), pp. 442-464. http://jou.sagepub .com/content/6/4/442.short [Consulta: 6 de mayo de 2014]

DEUZE, Mark. (2002): Journalists in the Netherlands. An analysis of the people, the issues and the (inter-) national environment. Tesis doctoral dirigida por el Dr. E.A. van Zoonen y el Dr. K. Schönbach, Amsterdam School of Communication Research (ASCOR) de la Universidad de Amsterdam, Biblioteca de la Universidad de Ámsterdam.

EEMEREN, Frans Hendrik van (2011): "In Context. Giving contextualization its rightful place in the study of argumentation". Argumentation, 25, pp. 141-164.

EEMEREN, Frans Hendrik van (ed) (2001): Crucial concepts in argumentation theory, Amsterdam, SicSat.

EEMEREN, Frans Hendrik van \& GROOTENDORST, Robert (2004): A systematic Theory of Argumentation. The pragma-dialectical approach. New York, Cambridge University Press.

EEMEREN Frans Hendrik van \& GROOTENDORST, Robert (ed., 1994): Studies in Pragma-Dialectics, Holanda, Sic Sat. International Centre for the Study of Argumentation.

EEMEREN, Frans Hendrik van; GROOTENDORST, Robert; JACKSON, Sally \& JACOBS, Scott (1993): Reconstructing Argumentative Discourse. USA, The University of Alabama Press.

EEMEREN, Frans Hendrik van; GROOTENDORST, Robert; \& SNOECK HENKEMANS, Francisca (2002): Argumentation. Analysis, Evaluation, Presentation. USA, Lawrence Erlbaum Associates, Inc.

EEMEREN, Frans Hendrik van; GROOTENDORST, Robert \& SNOECK-HENKEMANS, Francisca (1996): Fundamentals of Argumentation Theory. A handbook of historical background and contemporary developments. New Jersey, Lawrence Erlbaum Associates, Inc.

GÓMEZ MOMPART, Josep LLuís; GUTIÉRREZ LOZANO, Juan Francisco; y PALAU SAMPIO, Dolors (Eds., 2013): La calidad periodística. Teorías, investigaciones y sugerencias profesionales. Col·lección 'Aldea Global', n’ 26. Barcelona - Castelló - València, UAB, UJI, UPF y UV.

PARREÑO, Mònica (2013): "La argumentación de los periodistas sobre la calidad del periodismo". En GÓMEZ MOMPART, Josep L.; GUTIÉRREZ LOZANO, Juan Francisco; y PALAU SAMPIO, Dolors (Eds.). La calidad periodística. Teorías, investigaciones y sugerencias profesionales. Col·lecció 'Aldea Global', $\mathrm{n}^{\circ}$ 26. Barcelona - Castelló - València: UAB, UJI, UPF y UV. Pp. 105-118. 
PARREÑO, Mònica (2012): El camp periodístic i els professionals de la informació al País Valencià. Anàlisi de l'estructura comunicativa i de les argumentacions dels periodistes sobre el periodisme. Tesi de doctorat amb menció internacional. Dirigida por el Dr. Jesús Alcolea Banegas y por el Dr. Rafael Xambó i Olmos. Universitat de València. TESEO: https://www.educacion.gob.es/teseo/mostrarSeleccion.do\#.

SNOECK-HENKEMANS, Francisca (1997): Analyzing complex argumentation. The reconstruction of multiple and coordinatively compound argumentation in a critical discussion. Amsterdam, SicSat.

WILLARD, Charles Arthur (1989a): A theory of argumentation. Alabama, The University of Alabama Press.

WILLARD, Charles Arthur (1989b): “Argument as Social Enterprise”. En MAIER, Robert (Ed.): Norms in argumentation: proceedings of the Conference on Norms 1988. Dordrecht, Foris Publications.

WOODS, John (2006): "Pragma-Dialectics: A retrospective". En HOUTLOSSER, Peter \& REES, Agnes van: Considering Pragma-Dialectics. New Jersey, Lawrence Erlbaum Associates, Inc.

XAMBÓ, Rafael (2012): “The media in the Valencian Country". Catalan Journal of Communication and Media Studies, 1, vol. 4, pp. 107-122. 